\title{
VOLUME X
}

\section{INDEX OF SUBJECTS AND AUTHORS}

\author{
Only main topics are listed, and reference is given to the first page \\ of each paper in which they are discussed.
}

Acriflavine, effects on episome and bacterial chromosome transfer in Escherichia coli $\mathrm{K} 12,241$

Adams, M. S., 313

Aedes aegypti, comparative genetical study on DDT resistance, 219

Aegilops speltoides, homoeologous pairing of chromosomes from Agropyron elongatum, 63

Agropyron elongatum, homoeologous pairing of chromosomes from, with Aegilops speltoides and Triticum aestivum, 63

Anopheles gambiae, genetic studies on muta. tion in species $A$ and $B, 205$

Arai, T., 241

Aromatic amino acid synthesis in Aspergillus nidulans, 261

Ascobolus immersus, simultaneous conversion in linked genes, 121

Ascomycetes, differential maturation of asci and recombination, 1

Aspergillus nidulans

aromatic amino acid synthesis and parafluorophenylalanine resistance, 261

chromosome instability related to gene suppression, 173

Aspergillus niger, genetic analysis by means of parasexual cycle, 45

Autogamy, maintenance of heterozygosity after, in Euplotes, 35

Bacteriophages, polarized exclusion of $\mathrm{T} 2$ in crosses with T4, 13

Ball, C., 173

Bishop, J. O., 107

Canalized phenotype, selection for and against, 21

Cell number in relation to heterosis in em. bryonic growth in mouse, 235

Chloramphenicol, inhibition of respiratory enzyme synthesis by, in Saccharomyces cerevisiae, 199

Chromosome instability and gene suppression in Aspergillus nidulans, 173
Claxton, J. H., 161

Cleft palate in man in relation to developmental 'noise', 313

Colicin Ela, sex factor of, in Salmonella typhimurium, 323

Congenital tremor, penetrance of, in chick, 229

Conversion, simultaneous in linked genes in Ascobolus immersus, 121

Cytoplasmic mutant (SG), heterokaryon studies in Neurospora crassa, 185

Dawson, G. W. P., 127

Davison, J. R. N., 107

DD'T resistance, a genetical study in adult and larval Aedes aegypti, 219

De Groot, B., 13

Developmental 'noise' and a congenital malformation in man, 313

Differential maturation of asci and recombination studies, 1

Diuretic responses, selection for, in mouse, 95

Divergence in Vetukhiv's experimental populations of Drosophila pseudoobscura, 303

Drosophila

genetic divergence in Vetukhiv's experimental populations, 303

inversion heterozygosity and selection for wing length, 81

relationship between heterochromatiza. tion and variegation, 143

selection for and against canalized phenotype, 21

\section{Escherichia coli}

episome and chromosome transfer, effects of acriflavine, in $\mathrm{K} 12,241$

partial deletion map of the galactose operon of K12, 107

Euplotes minuta, maintenance of heterozygosity of $m t$ locus after autogamy, 35 
Finlayson, J. S., 195

Frame, R. M., 107

Galactose operon, partial deletion map of, in Escherichia coli K12, 107

Genetic analysis by the parasexual cycle in Aspergillus niger, 45

Genetic variation in diuretic responses in mouse, 95

Hair follicle development in tabby mice, 161

Hartmann-Goldstein, I. J., 143

Heterochromatization, relationship between, and variegation in Drosophila melanogaster, 143

Heterokaryon studies of a cytoplasmic mutant in Neurospora crassa, 185

Heterosis in relation to cell number in embryonic growth of mouse, 235

Homoeologous pairing of chromosomes from Agropyron elongatum with Triticum aestivum and Aegilops speltoides, 63

Hudson, D. M., 195

Hybrids between pigeons and doves and relationships amongst interaction products, 273

Inbreeding, effects on litter size in mouse, 73

Induced mating technique for Anopheles gambiae, 205

Interaction antigen in hybrids of pigeons and doves, 273

Inversion heterozygosity and selection for wing length in Drosophila melanogaster, 81

Irwin, M. R., 273

Jarolmen, H., 289

Johnson, R., 63

Kimber, G., 63, 295

Kitagawa, O., 303

Krzanowska, H., 235

LaBar, M. M., 273

Lamb, B. C., 1

Lawn, A. M., 323

Lhoas, P., 45

Linkage of a urinary protein component to brown coat colour locus in mouse, 195

Linnane, A. W., 199

Litter size, effects of inbreeding on, in mouse, 73

Luporini, P., 35
Maintenance of heterozygosity after autogamy in Euplotes, 35

Mason, G. F., 205

Maturity, genetic control of, in Tetrahymena pyriformis, 319

McCarthy, J. C., 73

Meynell, G. G., 323

Microtus agrestis, serum esterase variation, 135

Mouse

cell number in relation to heterosis during embryonic growth, 235

effects of inbreeding on litter size, 73

hair follicle development in tabby $(T a), 161$

linkage of a urinary protein component to brown coat colour locus, 195

Nijmegen waltzer, a new neurological mutant, 117

selection for diuretic responses, 95

\section{Neurospora crassa}

differential maturation of asci, 1

heterokaryon studies of a cytoplasmic mutant, 185

Neurospora, unbiased second division segregation, 289

Nicotiana, $\mathbf{S}$ gene polymorphism, 251

Nijmegen waltzer $(n v)$, a new neurological mutant in mouse, 117

Niswander, J. D., 313

Nobili, R., 35

Pandey, K. K., 251

Parasexual cycle, genetic analysis in Aspergillus niger, $\mathbf{4 5}$

Paszewska, A., 121

Penetrance of congenital tremor in White Leghorn chicks, 229

Polarized exclusion of T2 in crosses with T4, 13

Polymorphism of $\mathbf{S}$ gene in Nicotiana species and genotypes, 251

Potter, M., 195

Prevosti, A., 81

Puhalla, J. E., 185

Recombination effect on differential maturation of asci, 1 exclusion of $\mathrm{T} 2$ in crosses with $\mathrm{T} 4,13$

Resistance to para-fluorophenylalanine in Aspergillus nidulans, 261

Reversion of tryA in Salmonella typhimurium, 127

Riyasaty, S., 127

Russell, M. A., 135 
Saccharomyces cerevisiae, inhibition of respiratory enzyme synthesis by chloramphenicol, 199

Salmonella typhimurium

frequent try $A$ auxotrophs in, 127

sex factor of colicin $\mathrm{Ela}, 323$

Saunders, G., 199

Segregation, unbiased second-division, in Neurospora species, 289

Selection

for and against canalized phenotype in Drosophila, 21

for diuretic responses in mouse, 95

for wing length, and inversion heterozygosity in Drosophila subobscura, 81

Self-incompatability alleles in Nicotiana, 251

Semeonoff, R., 135

Serum esterase, variation in Microtus agerestis, 135

Simon, Ellen M., 319

Sinha, U., 261

Sittmann, K., 229

Sordaria fimicola, differential maturation of asci, 1

Spickett, S. G., 95

Srb, A. M., 185, 289

Stewart, J., 95

Suppression, chromosome instability and, in Aspergillus nidulans, 173
Tetrahymena pyriformis, genetic control of maturity, 319

Translocated alien chromosome segment to wheat chromosome, 295

Triticum aestivum

homoeologous pairing of chromosomes from Agropyron elongatum, 63

recognition of alien chromosome segment, 295

Urinary protein component linked to brown coat colour in mouse, 195

van Abeelen, J. H. F., 117

van der Kroon, P. H. W., 117

Variegation, relationship with heterochromatization, in Drosophila melanogaster, 143

Vetukhiv's experimental population of Drosophila pseudoobscura, genetic divergence in, 303

Watanabe, T., 241

Wheat, homoeologous pairing of chromosomes in complex hybrids, 63

Wilkie, D., 199

Wood, R. J., 219

Young, S. S. Y., 21 